\title{
EQUILÍBRIO DE ADSORÇÃO DO CORANTE RODAMINA B EM CARVÃO ATIVADO OBTIDO DOS RESÍDUOS DO COCO VERDE
}

\section{EQUILIBRIO DE ADSORCIÓN DEL COLORANTE RODAMINA B EN CARBÓN ACTIVADO OBTENIDO DE LOS RESIDUOS DEL COCO VERDE}

\author{
E. S. C. DE ALMEIDA E SILVA ${ }^{1}$, B. C. PINTO ${ }^{1}$, A. S. LIMA ${ }^{1}$, M. A. DE OLIVEIRA ${ }^{1}$, A. \\ C. DA SILVA BEZERRA ${ }^{2}$, T.S.F.SOUZA ${ }^{1}$, C.G. RODRIGUES ${ }^{1}$, A. R. T.MACHADO ${ }^{1,3}$. \\ ${ }^{1}$ Centro Universitário de Belo Horizonte, Instituto de Engenharia e Tecnologia, Brasil \\ ${ }^{2}$ Centro Federal de Educação Tecnológica de Minas Gerais, Departamento de Engenharia de \\ Transportes, Brasil \\ ${ }^{3}$ Universidade do Estado de Minas Gerais, Faculdade de Engenharia, Brasil \\ E-mail: estela.092@hotmail.com
}

\author{
article info \\ Article history: \\ Received 2017-09-04 \\ Accepted 2017-11-20 \\ Available online 2017-12-20
}

PALAVRAS-CHAVE: Adsorção; Carvão Ativado; Coco Verde; Rodamina B. PALABRAS-CLAVE: Adsorción; Carbón Activado; Coco Verde; Rodamina B.

RESUMO: Com o objetivo ao desenvolvimento de materiais alternativos para o tratamento de efluentes aquosos contendo contaminantes orgânicos, com este trabalho, foi produzido um carvão ativado utilizando como fonte de carbono os resíduos do coco verde. Ademais, o comportamento da adsorção do corante rodamina $B$, no material produzido, foi estudado em função da temperatura por meio das isotermas de adsorção e de seus parâmetros termodinâmicos. Os resultados obtidos mostraram que a adsorção da rodamina $B$ em carvão ativado é um processo espontâneo e endotérmico, e a quantidade de rodamina $B$ adsorvida aumenta com a concentração a uma temperatura constante. Os resultados demonstraram, também, o potencial dos resíduos do coco verde como matéria-prima para obtenção de carvão ativado, bem como para o tratamento de efluentes contendo contaminantes orgânicos.

RESUMEN: Con el fin de desarrollar materiales alternativos al tratamiento de efluentes acuosos conteniendo contaminantes orgánicos, fue producido, con este trabajo, un carbón activado utilizando como fuente de carbón los residuos de cocos verdes. Además, el comportamiento de la adsorción del colorante rodamina B, en el material producido, fue estudiado en función de la temperatura por medio de las isotermas de adsorción y sus parámetros termodinámicos. Los resultados obtenidos mostraron que la adsorción de la rodamina $B$ en carbón activado es un proceso espontáneo y endotérmico, y la cantidad de rodamina $B$ adsorbida aumenta con la concentración en una temperatura constante. Los resultados demostraron, además, el potencial de los residuos del coco verde como materia prima para la obtención de carbón activado, así como para el tratamiento de efluentes conteniendo contaminantes orgánicos. 


\section{INTRODUÇÃO}

O carvão ativado é usado em processos em que se deseja remover determinadas substâncias de um fluido, por meio do fenômeno da adsorção. Esse produto é um material carbonáceo e poroso, preparado pela pirólise de substâncias orgânicas, principalmente, de origem vegetal. A sua capacidade adsorvente é proveniente da alta área superficial e da presença de uma variedade de grupos funcionais em sua superfície (MULLER et al., 2009).

A produção de carvão ativado no Brasil mostra-se insuficiente frente às suas reais necessidades e, também porque apresenta um mercado consumidor promissor, envolvendo pequenas, médias e grandes empresas (BORGES et al., 2003). Assim, inúmeros processos fazem uso do carvão ativado, por exemplo, para purificação e clarificação de alimentos, fabricação de medicamentos, tratamento de ar, tratamento de água, tratamento de efluentes, catálise, entre outros (PEREIRA, 2010).

O carvão ativado pode ser produzido a partir de matéria-prima de baixo custo, por exemplo, a casca de coco verde, que se torna um resíduo após o consumo da água de coco (SCHETTINO JR et al., 2007). Desta forma, o processo de remoção de contaminantes orgânicos por adsorção, torna-se economicamente viável (SCHETTINO JR et al., 2007), uma vez que o carvão ativado possui uma alta adsortibilidade de compostos orgânicos (CASTRO, et al., 2009). Neste contexto, em Belo Horizonte, o resíduo de coco verde apresenta-se como opção atraente para obtenção de carvão ativado, uma vez que apenas na orla da Lagoa da Pampulha são recolhidas, em média, 30 toneladas de carcaça de cocos aos finais de semana.

Com este trabalho, foi produzido um carvão ativado utilizando a casca do coco verde como matéria-prima. Além disso, foi realizado um estudo de adsorção, utilizando como modelo soluções aquosas do corante catiônico rodamina B (Figura 1). Os dados de equilíbrio sólido-líquido na forma de isotermas de adsorção são apresentados.<smiles>CCN(CC)c1ccc2c(-c3ccccc3C(=O)O)c3ccc(=[N+](CC)CC)cc-3oc2c1</smiles>

Figura 1 - Fórmula estrutural do corante rodamina B. 


\section{PROCEDIMENTOS EXPERIMENTAIS}

\subsection{Preparo do carvão ativado}

As carcaças de cocos foram coletadas nos arredores da Lagoa da Pampulha, em Belo Horizonte, Minas Gerais. Para a produção do carvão ativado, as cascas desses resíduos foram desfiadas e lavadas com água quente, até que a água de lavagem se tornasse límpida. Em seguida, o material obtido foi seco em estufa a $105^{\circ} \mathrm{C}$ e triturado em liquidificador doméstico. $500 \mathrm{~g}$ do material, processado, foram submetidos à ativação com ácido fosfórico. Para este experimento, foi adicionado ao material precursor $500 \mathrm{~mL}$ do ácido e, agitou-se manualmente por $3 \mathrm{~min}$. A mistura resultante foi lavada com água destilada e submetida à carbonização em forno mufla a $350{ }^{\circ} \mathrm{C}$ por $60 \mathrm{~min}$. Após resfriamento, lavou-se o material com uma solução de bicarbonato de sódio $1 \%(\mathrm{~m} / \mathrm{v})$, seguido de lavagem com água destilada e secagem em estufa a $105^{\circ} \mathrm{C}$.

\subsection{Caracterização do carvão produzido}

O carvão obtido foi caracterizado pelas de técnicas de microscopia de varredura (MEV) em um microscópio eletrônico de varredura da marca HITASHI, modelo TM 3000, com detectores de elétrons retroespalhados.

\subsection{Ensaios de adsorção}

$100 \mathrm{mg}$ do carvão ativado foi transferido para um erlenmeyer. Em seguida, foram adicionados $25 \mathrm{~mL}$ da solução aquosa de rodamina $\mathrm{B}\left(2,5 \mathrm{mmol} \mathrm{L}^{-1}\right)$. Esse procedimento foi repetido com soluções nas concentrações de 0,$2 ; 0,4 ; 0,8 ; 1,2$ e $1,7\left(\mathrm{mmol} \mathrm{L}^{-1}\right)$. Os ensaios foram realizados à temperatura de $25^{\circ} \mathrm{C}, \mathrm{o} \mathrm{pH}$ foi mantido no $\mathrm{pH}$ normal da solução e, os erlenmeyers mantidos sob agitação, em Shaker a $150 \mathrm{rpm}$ por $24 \mathrm{~h}$. Após esse período, a mistura foi centrifugada e, a concentração remanescente monitorada por espectroscopia de Uv-Vís, no comprimento de onda de $550 \mathrm{~nm}$. A quantidade de rodamina B adsorvida por unidade de massa do adsorvente $\left(\mu \mathrm{mol} \mathrm{g}^{-1}\right)$ foi calculada usando-se a Equação 1(NASCIMENTO et al., 2014).

$$
q_{e}=\frac{\left(C_{i}-C_{e}\right)}{m} \times V
$$

Em que $C_{i}$ e $C_{e}$ são as concentrações inicial e final na fase líquida (mmol L-1), respectivamente, $\mathrm{m}$ é a massa de adsorvente $(\mathrm{g})$ e $\mathrm{V}$ é o volume da solução (L).

A influência da temperatura no processo de adsorção foi avaliada, variando as temperaturas de adsorção $\left(35,45\right.$ e $\left.55^{\circ} \mathrm{C}\right)$. 


\subsection{Modelos de adsorção}

Os dados das isotermas de equilíbrio foram ajustados segundo os modelos de Langmuir e Freundlich, Equações 2 e 3 (NASCIMENTO et al., 2014), respectivamente.

$$
\begin{aligned}
& \frac{q_{e}}{q_{\max }}=K_{L} x \frac{C_{e}}{1+K_{L} C_{e}} \\
& q_{e}=K_{F} C_{e}^{1 / n}
\end{aligned}
$$

De acordo com as equações $q_{\max }$ é a capacidade máxima de adsorção $\left(\mathrm{mol} \mathrm{g}^{-1}\right), K_{L}(\mathrm{~L}$ $\left.\mathrm{mol}^{-1}\right)$ é a constante de Langmuir, $K_{F}\left(\mathrm{~mol} \mathrm{~g}^{-1}\right)$ e $\mathrm{n}$ são constantes de Freundlich e, $C_{e}$ é a concentração do adsorvato após o equilíbrio ser alcançado.

As formas lineares, das isotermas de Langmuir e Freundlich, Equações 4 e 5, respectivamente, foram empregadas para determinação dos valores das constantes dos respectivos modelos (NASCIMENTO et al., 2014).

$$
\begin{aligned}
& \frac{C_{e}}{q_{e}}=\frac{1}{q_{\max } x K_{L}}+\frac{C_{e}}{q_{\max }} \\
& \log q_{e}=\log K_{F}+\frac{1}{n} \log C_{e}
\end{aligned}
$$

\subsection{Parâmetros termodinâmicos}

Os parâmetros termodinâmicos, entalpia $\left(\Delta_{a d s} H\right)$, entropia $\left(\Delta_{a d s} S\right)$ e energia Livre de Gibbs $\left(\Delta_{a d s} G\right)$, foram obtidos utilizando as Equações 6 e 7 (NASCIMENTO et al., 2014).

$$
\begin{aligned}
& \Delta_{a d s} G=\Delta_{a d s} H-T \Delta_{a d s} S \\
& \ln K_{L}=\frac{\Delta_{a d s} S}{R}-\frac{\Delta_{a d s} H}{R T}
\end{aligned}
$$

Em que:

$R$ é a constante universal dos gases $\left(\mathrm{J} \mathrm{K}^{-1} \mathrm{~mol}^{-1}\right) ; T$ é a temperatura em Kelvin $(\mathrm{K})$ e $K_{\mathrm{L}}$ é a constante de Langmuir( $\left(\mathrm{L} \mathrm{mol}^{-1}\right)$.

\section{RESULTADOS E DISCUSSÃO}

As micrografias do precursor mostraram um material com estrutura fibrosa (Figuras 2a e 2b). E, após o processo de carbonização, observa-se qualitativamente a formação de uma estrutura microporosa (Figuras $2 \mathrm{c}$ e $2 \mathrm{~d}$ ). No entanto, essa porosidade, aparentemente, não foi bem desenvolvida, ou ainda os poros podem estar obstruídos por materiais de decomposição 
formados durante a carbonização (BANSAL e GOYAL, 2005).

Os resultados experimentais da adsorção da rodamina B estão apresentados na Figura 3. Observa-se que o aumento da temperatura proporciona um aumento da quantidade adsorvida no equilíbrio, característica de um processo endotérmico. Neste processo, o aumento da temperatura contribui para uma adsorção mais alta, enquanto que nos processos exotérmicos a adsorção aumenta com a redução da temperatura (NASCIMENTO et al., 2014). Também vale a pena destacar, que os valores encontrados para $\mathrm{q}_{\mathrm{e}}$ foram superiores aos relatados em outros estudos (ARIVOLI et al., 2009).
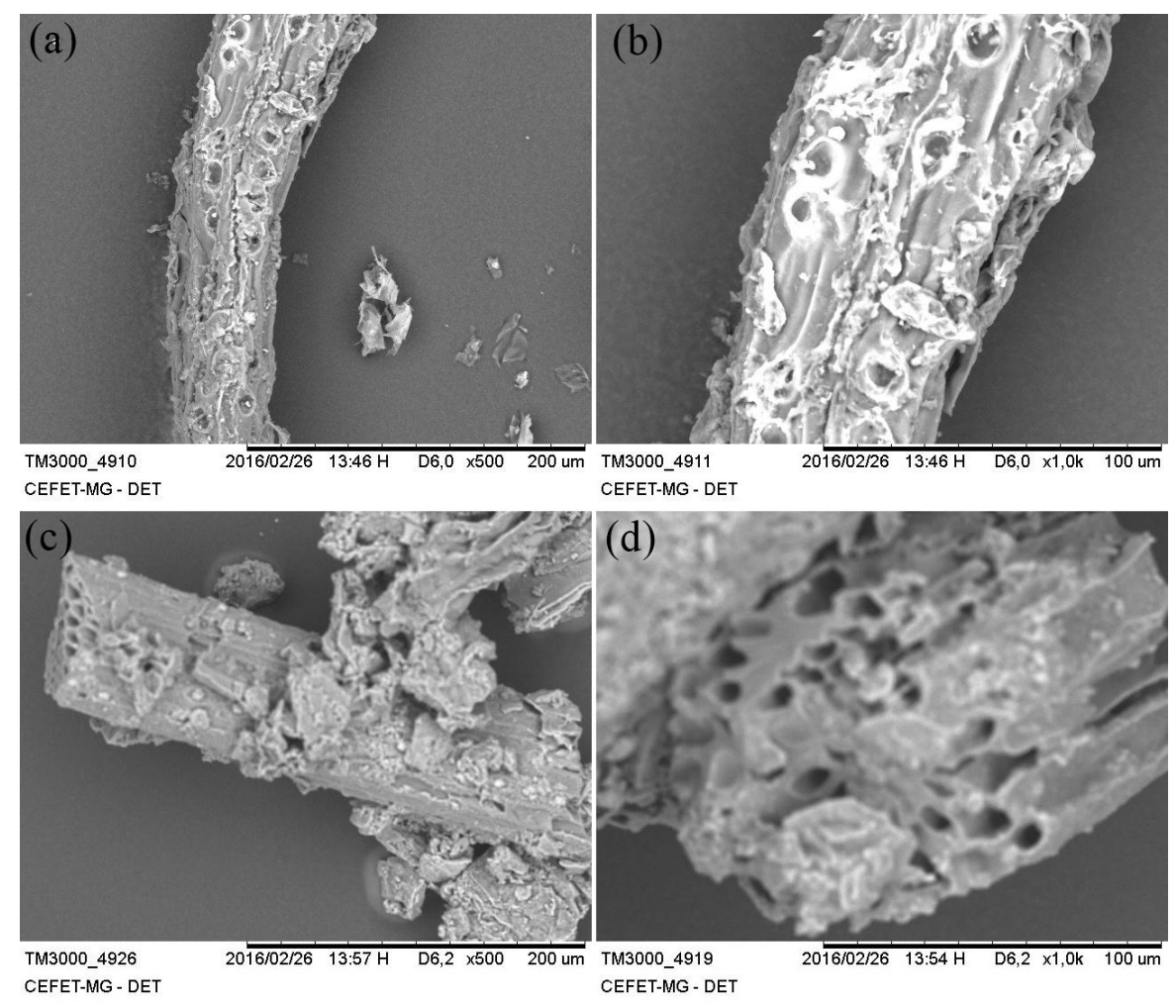

Figura 2- Micrografias obtidas em MEV. (a) e (b) do resíduo do coco verde com aumento de 500 e 1000 vezes, respectivamente. (c) e (d) do carvão ativado com aumento de 500 e 1000 vezes, respectivamente. 


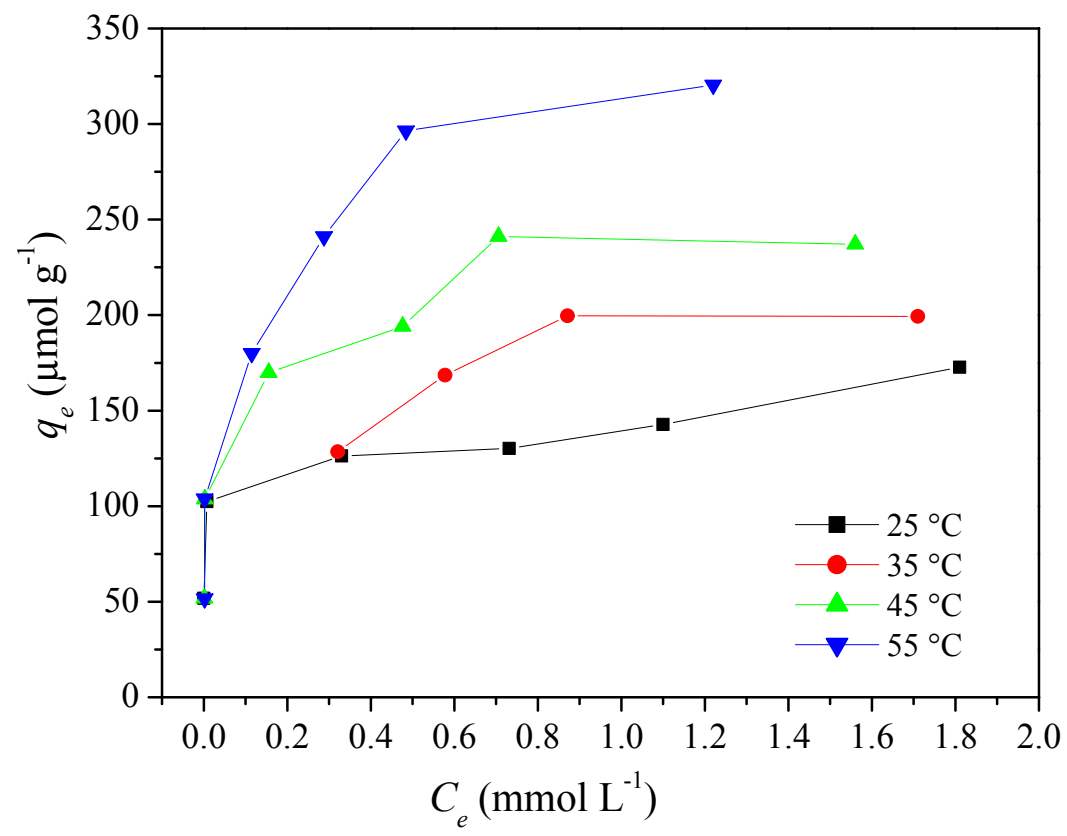

Figura 3- Isotermas de adsorção da rodamina B em carvão ativado produzido a partir do resíduo do coco verde.

Os dados de equilíbrio sólido-líquido foram ajustados de acordo com os modelos de isotermas de Langmuir e Freundlich. Os parâmetros obtidos aplicando-se esses modelos aos dados experimentais são apresentados na Tabela 1. Os resultados mostram que o modelo de isoterma de Langmuir apresentou melhor ajuste dos dados experimentais, uma vez que os coeficientes de correlação (R), obtidos pela linearização da equação, foram superiores aqueles encontrados com o modelo de isoterma de Freundlich (Tabela 1). Esses resultados sugerem um mecanismo de adsorção em superfícies homogêneas (Figura 4) com a superfície do adsorvente uniforme e, com todos os sítios ativos de adsorção possuindo a mesma energia (NASCIMENTO et al., 2014). Além disso, é possível sugerir uma adsorção ocorrendo em monocamadas, com capacidade máxima de adsorção, a $25^{\circ} \mathrm{C}$, de $168,03 \mu$ mol rodamina $\mathrm{B} / \mathrm{g}$ de carvão ativado.

Tabela 1 - Parâmetros de Langmuir e Freundlich para adsorção da rodamina B em carvão ativado obtido do resíduo do coco verde

\begin{tabular}{|c|c|c|c|c|c|c|}
\hline \multirow[b]{2}{*}{ Temperatura $\left({ }^{\circ} \mathrm{C}\right)$} & \multicolumn{3}{|c|}{ Modelo de Langmuir } & \multicolumn{3}{|c|}{ Modelo de Freundlich } \\
\hline & $\begin{array}{c}q_{\max } \\
\left.(\mu \mathrm{mol} \mathrm{g})^{-1}\right)\end{array}$ & $\begin{array}{c}K_{L} \\
\left(\mathrm{~L} \mathrm{~mol}^{-1}\right) \\
\end{array}$ & $\mathrm{R}^{2}$ & $\begin{array}{c}K_{F} \\
\left.(\mu \mathrm{mol} \mathrm{g})^{-1}\right)\end{array}$ & $\mathrm{n}$ & $\mathrm{R}^{2}$ \\
\hline 25 & 168,03 & $1,26 \times 10^{4}$ & 0,99 & 370,47 & 7,55 & 0,93 \\
\hline 35 & 204,23 & $1,58 \times 10^{4}$ & 0,99 & 483,64 & 7,02 & 0,88 \\
\hline 45 & 240,86 & $2,82 \times 10^{4}$ & 0,99 & 864,63 & 5,30 & 0,95 \\
\hline 55 & 328,81 & $1,99 \times 10^{4}$ & 0,99 & $1.659,89$ & 4,20 & 0,94 \\
\hline
\end{tabular}




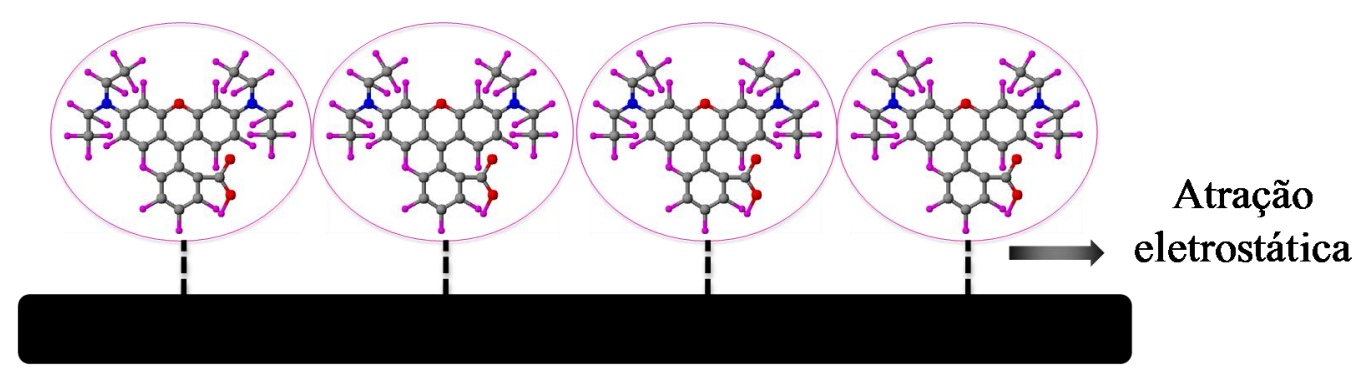

Superfície do carvão ativado

Figura 4- Esquema da adsorção em monocamada.

Os parâmetros termodinâmicos, energia livre de Gibbs $\left(\Delta_{a d s} G\right)$, entalpia $\left(\Delta_{a d s} H\right)$, e entropia $\left(\Delta_{a d s} S\right)$ são os indicadores verdadeiros da aplicação prática do processo de adsorção. Os resultados para esses parâmetros da adsorção do corante rodamina B são apresentados na Tabela 2. Os resultados da $\Delta_{a d s} G$ foram negativos em todas as temperaturas, confirmando a natureza espontânea da adsorção. Ademais, vale ressaltar que quanto mais negativo o valor de $\Delta_{a d s} G$, mais energeticamente favorável é o processo. Valores reduzidos de $\Delta_{a d s} G$ implicam em uma maior força motriz para o processo de adsorção (ARIVOLI et al., 2009). O sinal positivo de $\Delta_{a d s} H$ indica que o processo de adsorção possui caráter endotérmico. Além disso, os valores são da ordem de grandeza de adsorção do tipo física, indicando presença de interações eletrostáticas (ARIVOLI et al., 2009). O valor positivo para $\Delta_{a d s} S$ indica que há um aumento do grau de liberdade no sistema. Destaca-se ainda que devido ao fato do termo $T x \triangle_{a d s} S$ apresentar um valor maior que $\Delta_{a d s} H$ para todas as temperaturas avaliadas, acredita-se que o aumento do grau de liberdade na interface sólido-líquido é o que guia a adsorção, isto é, um processo entropicamente dirigido (MARTINEZ SAAVEDRA, 2016).

Tabela 2 - Parâmetros termodinâmicos para adsorção da rodamina B em carvão ativado obtido do resíduo do coco verde

\begin{tabular}{crrr}
\hline Temperatura $\left({ }^{\circ} \mathrm{C}\right)$ & $\Delta_{a d s} G\left(\mathrm{~kJ} \mathrm{~mol}^{-1}\right)$ & $\Delta_{a d s} H\left(\mathrm{~kJ} \mathrm{~mol}^{-1}\right)$ & $\Delta_{a d s} S\left(\mathrm{~kJ} \mathrm{~mol}^{-1} \mathrm{~K}^{-1}\right)$ \\
\cline { 1 - 2 } 25 & $-23,361$ & & \\
\cline { 1 - 2 } 35 & $-56,699$ & 31,498 & 0,184 \\
\cline { 1 - 2 } 45 & $-58,539$ & & \\
\cline { 1 - 2 } 55 & $-60,379$ & & \\
\hline
\end{tabular}

\section{CONCLUSÃO}

O equilíbrio de adsorção foi melhor descrito pela isoterma de Langmuir. Os resultados demonstram que adsorção é de natureza endotérmica, os valores de $\Delta_{a d s} G$ obtidos confirmaram a espontaneidade da adsorção e o valor de $\Delta_{a d s} H$ é da ordem de fisissorção. Já o valor positivo de $\Delta_{a d s} S$ indica que há um aumento na interface sólido-fluido com o aumento da temperatura. O carvão ativado obtido apresenta potencial para remoção de corantes orgânicos. 


\section{AGRADECIMENTO}

Os autores agradecem À FAPEMIG (Fundação de Amparo à Pesquisa do Estado de Minas Gerais), pelo apoio financeiro.

\section{REFERÊNCIAS}

ARIVOLI, S.; THENKUZHALI, M.; DEVAPRASATH, P.M. Adsorption of Rhodamine B by acid activated carbon-kinetic, thermodynamic and equilibrium studies. Orbital, v.1, n.2, p.138-155, 2009.

BANSAL, R.C.; GOYAL, M. Activated carbon adsorption. Flórida, USA: Taylor e Francis Group - Boca Raton,2005.

BORGES, F. M.; SILVA, F. P.; CARVALHO, M. C.; FILHO, A. G. Desenvolvimento e criação de uma unidade produtiva de carvão ativado. In: ENCONTRO NACIONAL DE ENGENHARIA DE PRODUÇÃO, 2003. Ouro Preto. Anais eletrônicos... Ouro Preto: Associação Brasileira de Engenharia de Produção (ABEPRO), 2003. 8 p. Disponível

em $<$ http://www.abepro.org.br/biblioteca/ENEGEP2003_TR0502_0601.pdf >. Acesso em 07/09/2017.

CASTRO, C.S.; GUERREIRO, M.C.; OLIVEIRA, L.C. A.; GONÇALVES, M. Remoção de compostos orgânicos em água empregando carvão ativado impregnado com óxido de ferro: ação combinada de adsorção e oxidação em presença de $\mathrm{H}_{2} \mathrm{O}_{2}$. Química Nova, São Paulo, SP, v.32, n.6, p.1561-1565, 2009.

MARTINEZ SAAVEDRA, L. N. Sínteses de polímeros molecularmente impressos para adsorção seletiva de Quinolina em matriz orgânica. 2017. 129 f. Dissertação (Mestrado em Química) - Universidade Federal de Ouro Preto, Ouro Preto, MG, 2016.

MÜLLER, C. C.; RAYA-RODRIGUEZ, M. T.; AND CYBIS, L. F. Adsorção em carvão ativado em pó para remoção de microcistina de água de abastecimento público. Eng. Sanit. Ambient., Rio de Janeiro, RJ, v. 14, n. 1, p. 29-38, mar. 2009. Disponível em $\quad<$ http://www.scielo.br/scielo.php?script=sci_arttext\&pid=S1413$41522009000100004 \& \operatorname{lng}=$ pt\&nrm=iso $>$. Acesso em 07 Set. 2017.

NASCIMENTO, R. F. DO.; LIMA, A. C. A. DE.; VIDAL, C. B.; MELO, D. DE. Q.; RAULINO, G. S. C. Adsorção: aspectos teóricos e aplicações ambientais. Fortaleza: Imprensa Universitária, 2014.

PEREIRA, E. I. Produção de Carvão ativado a partir de diferentes percursores utilizando $\mathrm{FeCl}_{3}$ como agente ativante. 2010. 72 f. Dissertação (Mestrado em Agroquímica) - Universidade Federal de Lavras, Lavras, MG, 2010.

SCHETTINO JR., M. A.; FREITAS, J.C.C.; CUNHA, A.G.; EMMERICH, F.G.; SOARES, A.B.; SILVA, P.R. N. Preparação e caracterização de carvão ativado quimicamente a partir da casca de arroz. Química Nova, São Paulo, SP, v. 30, n.7, p.1663-1668, 2007. 